\title{
Switched-Beam Antenna for WSN Nodes Enabling Hardware-driven Power Saving
}

\author{
Luca Catarinucci, Sergio Guglielmi, Riccardo Colella, Luciano Tarricone \\ Department of Innovation Engineering, University of Salento \\ via per Monteroni, 73100, Lecce, Italy \\ Email: \{luca.catarinucci, sergio.guglielmi, riccardo.colella, luciano.tarricone\}@unisalento.it
}

\begin{abstract}
Energy saving is one of the most important issues in Wireless Sensor Network (WSN) context. Since the communication task is the most power-consuming operation, it is quite important to achieve an energy efficient communication in order to increase the lifetime of the devices through an intelligent use of the power transmission. In this context, the integration of WSN nodes with switched-beam antennas is becoming more and more appealing due to the possibility to extend sensor node lifetime by optimizing the transmitted power.

In this work a switched-beam antenna for WSNs nodes in the ISM band $(2.4-2.4835 \mathrm{GHz})$ is proposed. The radiating structure consists of four identical antennas, composed of an array of two L-shaped quarter-wavelength slot antenna elements arranged in a compact and symmetrical planar structure. Thanks to a properly designed switching circuit which controls the feeding of the antenna elements, one among eight possible different radiation patterns in the azimuth plane can be selected on the basis of specific needs. Simulations and experimental results, referred to a prototype realized on a FR-4 substrate, demonstrate the appropriateness of the proposed switched-beam antenna system as hardware element enabling new power saving strategies in WSN contexts.
\end{abstract}

\section{INTRODUCTION}

G UARANTEEING adequate energy efficiency in Wireless Sensor Networks (WSN) contexts is still an open issue. Indeed, WSN nodes, hereafter referred as motes, are frequently placed in unpractical or hardly accessible places and it could be difficult, time-consuming and expensive to replace batteries. Therefore, both WSN devices and communication protocols must be carefully designed to maximize the motes lifetime, in order to reduce maintenance costs and outages. In such a context, it is well known that the data communication through the RF frontend represents the most power consuming task [1-3]. Just to give an idea, the power required by the mote processor to process thousands of operations is comparable to that the RF transceiver needs to transmit a single bit. Therefore, in the last years, many techniques aimed at minimizing the energy consumption have been proposed [4-6]. Such techniques are often based on specific protocols optimizing the data transmission, for instance by periodically turning motes into sleep mode or opportunely controlling the radio transceiver activation/deactivation.

In order to further increase the energy efficiency, thereby extending even more the motes lifetime, the use of oppor- tunely controlled directional or switched-beam antennas could be a winning approach. Indeed, as already stated, motes are usually equipped with almost omnidirectional antennas and, consequently, only the power portion transmitted toward the proper mote is effectively used, whilst most of the power is wasted elsewhere. Vice versa, a mote provided with a switched-beam antenna could smartly reconfigure the antenna radiation pattern so to convey the power only toward the destination mote.

In this work a full planar and really compact switchedbeam antenna particularly suitable for WSN applications in the ISM band is proposed. When connected to the wireless module of a mote, it works in place of the common omnidirectional antenna and its radiation properties can be controlled through a digital interface. The proposed solution consists of four identical radiating structures, each one composed of two L-shaped quarter-wavelength slot antenna elements, arranged in a compact and symmetrical planar structure. Eight switchable radiation patterns with remarkable gain can be obtained, covering symmetrically 360 degrees in the azimuth plane, in order to reduce the transmission power and consequently extend the lifetime of the mote.

The paper is structured as follows: in Section II the state of the art on switched beam antennas in WSN context is discussed, whilst in Section III both working principle and design of the proposed antenna are described; later on in Section IV simulated and experimental results are shown and discussed. Finally, conclusions are drawn in Section V.

\section{Related Works}

Over the last few years, a strong research effort has been dedicated to the design of more and more performing switched-beam antennas [7-14]. Nevertheless, their integration in WSN motes to reduce energy consumption and to extend motes lifetime, has not been exhaustively explored yet. In particular, a smart switched-beam directional antenna is proposed in [7]. It is composed of four planar patch antennas arranged in a box-like structure. It can switch among four radiation beams with a uniform coverage of the azimuth plane and a good radiation gain in the main lobe direction, but its very large size is not compatible with the integration in WSN nodes. A pattern reconfigurable antenna composed of microstrip parasitic array elements is proposed in [8]. It is 


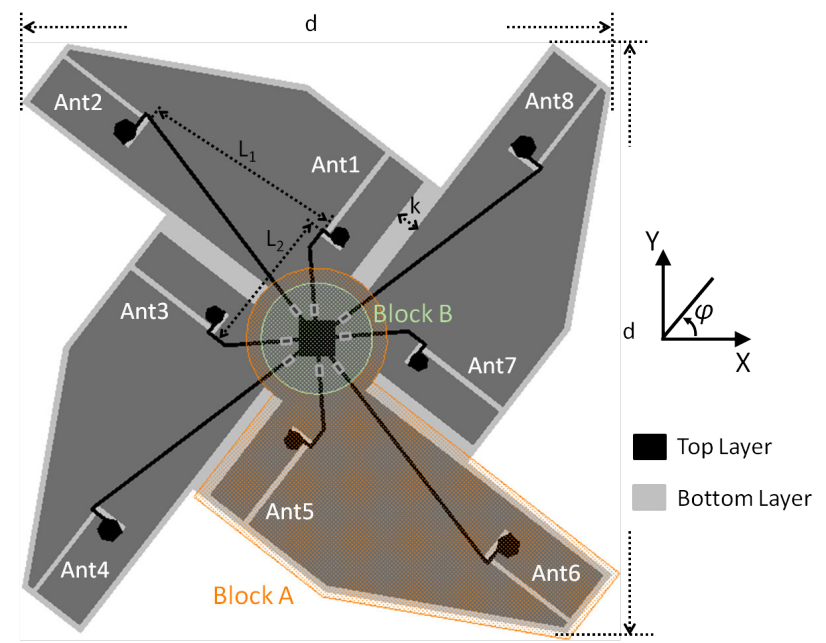

Fig 1. Structure of the proposed switched-beam antenna. The detailed structure of block A is shown in Fig. 2 and those of Block B in Fig. 3.

based on a simple and compact structure, but it does not ensure a 360-degree uniform coverage in the azimuth plane. Another interesting reconfigurable antenna for WSN sink nodes capable to switch between front-directional and conical beam pattern, is proposed in [9]. It is composed of an EBG ground plane and a power divider with a stub switched phase shifter. Despite the appreciable peak gain in the main lobe direction, it is rather cumbersome and presents no radiating beams in the azimuth plane. A reconfigurable angular diversity antenna using quad corners as reflector arrays and a switching control circuit is proposed in [10]. It presents a high radiation gain, but occupies a large volume and does not guarantee a uniform radiation patterns. On the contrary, a compact Switched-Beam Antenna composed of a four-element antenna array is presented in [11]. It shows eight switchable directional patterns and an omnidirectional one, thus ensuring an uniform coverage of the 360 degrees horizon. Moreover, it is both compact and inexpensive. Unfortunately, it exhibits an Half-Power Beam Width (HPBW) of a single beam of nearly 120 degrees, which causes a large overlapping area, thus not ensuring an optimized energy saving.

Finally, in our earlier work [12]-[14], a reconfigurable beam-steering antenna for WSN nodes is presented. It is composed of a vertical half-wavelength dipole antenna and eight microstrip antennas arranged in a $3 \mathrm{D}$ configuration. Thanks to a digital control circuit, it can switch among nine radiation patterns, one omnidirectional and eight directional with a HPBW of nearly 60 degrees in the azimuth plane. It guarantees a uniform coverage of the 360 degree horizon and a remarkable peak gain. The main issue is related to its integrability with real WSN nodes due to its 3D configuration.

In the following Sections, a new switched beam antenna controllable by and easily integrable into WSN nodes will be exhaustively presented along with simulated and measurement results.

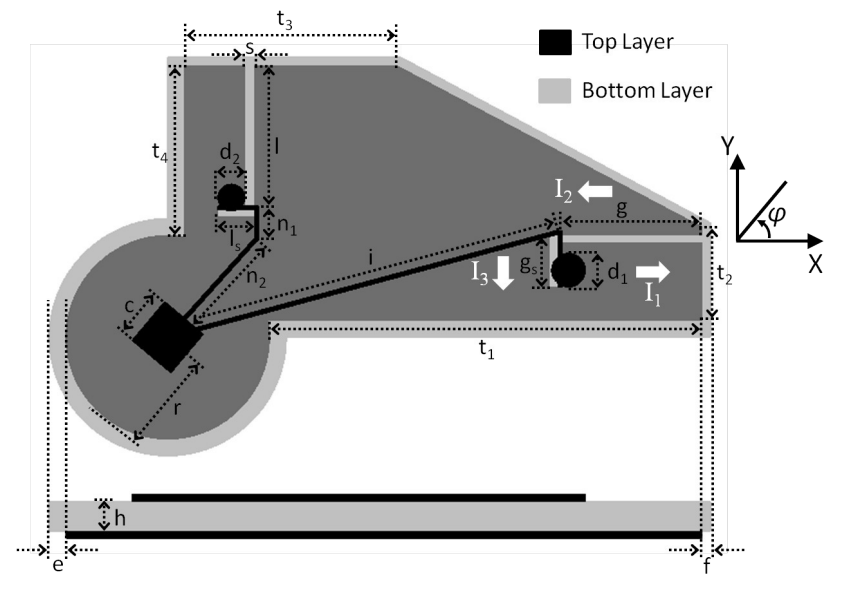

Fig 2. Geometry of the proposed array of two L-shaped quarter-wavelength slot antenna elements, corresponding to Block A in Fig. 1.

\section{Switched-Beam Antenna Design}

The overall structure of the proposed switched-beam antenna is shown in Fig. 1. In order to explain the characteristics of the designed radiating structure, the geometry of the array of two L-shaped quarter-wavelength slot antenna elements is firstly introduced.

The geometry of the proposed array of two L-shaped quarter-wavelength slot antenna elements is shown in Fig. 2. As described in [11], [15]-[16], a conventional L-slot antenna is composed of two slots connected at their ends with an angle of 90 degrees, with a total slot length near to a quarter-wavelength at the reference frequency. The antenna is fed by a $50 \Omega$ microstrip transmission line on the opposite side of the substrate (FR-4 in our case) with respect to the slot antenna. Such a feeding line configuration does not impact on weight and size of the entire system and it is suitable for the integration with electronic devices. As shown in Fig. 2 , the total current on the ground plane can be decomposed in three parts; in particular, currents $I_{1}$ and $I_{2}$ have opposite directions and therefore their contribution vanishes, leaving only the contribution of current $I_{3}$. Therefore, the L-shaped slot antenna works as a small dipole oriented in the $y$-axis, with a bidirectional pattern in the $x y$-plane. Furthermore, due to the shape of the ground plane that has a larger area in the direction of the feed point, the antenna presents a directional pattern in $x y$-plane, with a main lobe direction oriented toward $x$-axis. Typically, the L-shaped slot antenna exhibits a gain in the maximum radiation direction similar to that of a dipole.

The proposed array consists of two L-shaped quarterwavelength slot antenna elements, arranged at a distance of about a quarter of the wavelength at the reference frequency and perpendicular to each other. The resulting radiation pattern rotates of about 45 degrees from $x$-axis in the azimuth plane and the gain in the main lobe direction is about 5.09 $\mathrm{dBi}$. The greater gain with respect to a dipole antenna gives the possibility to reduce the transmission power and hence extend the lifetime of a mote. The proposed array architec- 

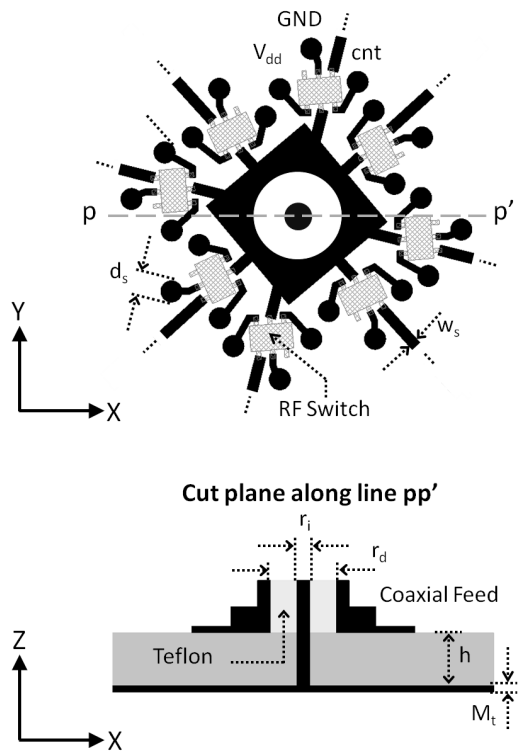

Fig 3. Detailed structure of the proposed switched-beam antenna, corresponding to Block B in Fig. 1.

ture is a good solution to increase energy efficiency or to reduce multipath effects, but it may deteriorate network performance if the signal comes from the directions of the nulls. For this reason, some arrays have been combined in order to have a uniform coverage of the 360 degrees in the azimuth plane.

The whole antenna structure is shown in Fig. 1 whilst the detailed parts, Block A and Block B, are depicted in Fig. 2 and 3 respectively. The antenna consists of four arrays of two L-shaped quarter-wavelength slot antenna elements arranged in a symmetrical planar structure. Basically, the array is replicated three times and rotated 90 degrees along the axis perpendicular to the $x y$-axis and passing through the center of the circumference with radius $r$. The radiating structure is fed by a $50 \Omega$ SMA connector at the center of the antenna, so that the feeding point is sufficiently far from the radiating elements and does not interact with them. The eight microstrip transmission lines are interrupted by RF switches that control the feeding of the antenna elements. In Fig. 3, the structure of the feeding point and the digital circuit that controls the RF switches are shown. Powered through the lines $V_{d d}$ and $G N D$, the state of the RF switches is controlled by the signal cnt; when cnt is set to $3 \mathrm{~V}$, the microstrip line is connected and the relative antenna element radiates, otherwise when it is set to $0 \mathrm{~V}$ the switch opens the line.

As described above, the array of two L-shaped quarterwavelength slot antenna elements exhibits a directional radiation pattern with the main lobe direction oriented as the bisector of the angle identified by the two antenna elements. Thanks to the symmetrical replication of the array, the antenna can illuminate a specific direction that is $n \pi / 2$ in the azimuth plane, where $n=0, \ldots, 3$. Moreover, feeding two non-adjacent L-shaped elements (in particular Ant1-Ant3,

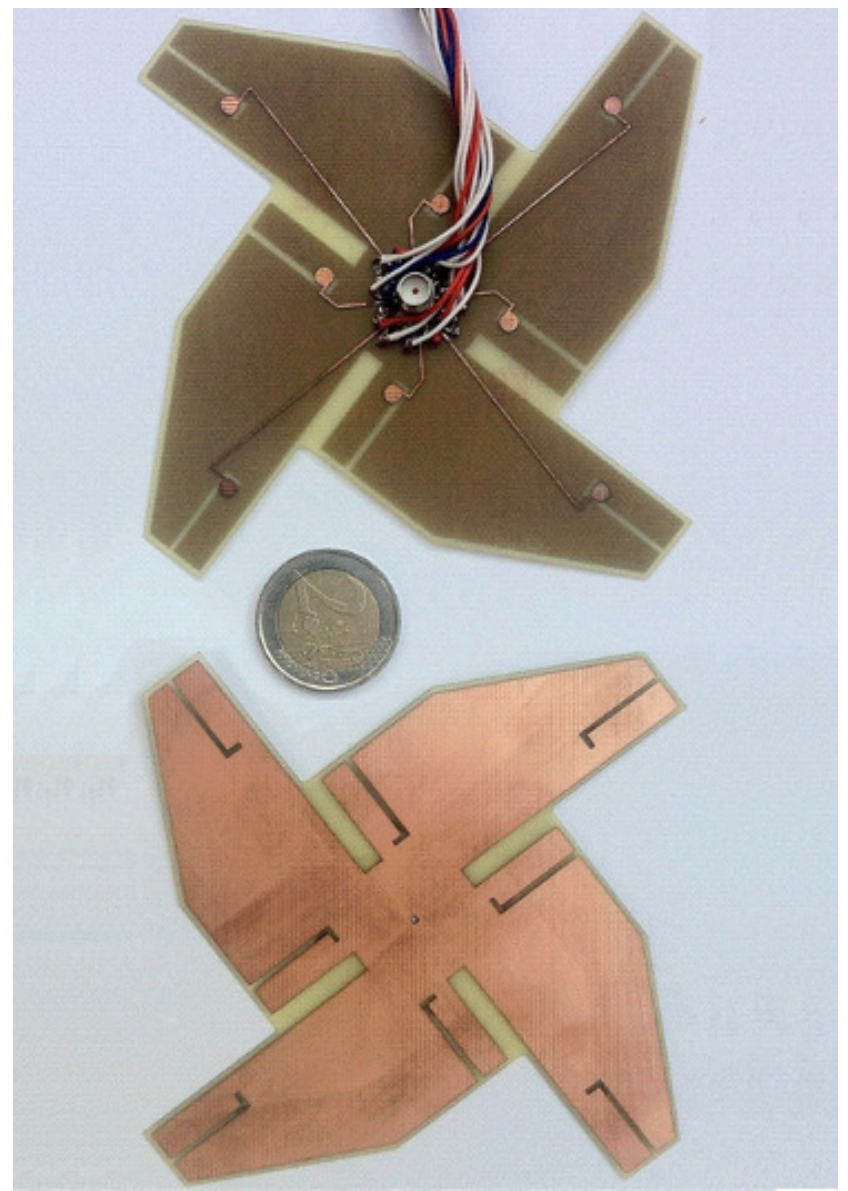

Fig 4. Proposed switched-beam antenna prototype (both sides).

Ant3-Ant5, Ant5-Ant-7 and Ant7-Ant1) other four patterns toward the directions $(1+2 n) \pi / 4$, where $n=0, \ldots, 3$, can be obtained. Therefore, by carefully setting the cnt control line of the RF switches, the proposed antenna can switch among eight symmetrical radiation patterns spaced 45 degrees from each other.

\section{Simulated and Experimental Results}

Several tests and measures have been performed in order to obtain an accurate characterization of the electromagnetic properties of the proposed antenna; for this purpose, a very cost-effective prototype of the switched-beam antenna with an overall size of $10 \times 10 \mathrm{~cm}^{2}$ has been fabricated through a technique described in [17]-[18], using an FR-4 substrate with thickness $M_{t}=0,8 \mathrm{~mm}$ and dielectric constant $\varepsilon_{r}=4,7$ (a) 2,45 GHz (see Fig. 4). The detailed design parameters of the proposed switched-beam antenna are listed in Table I. The used switches are the Peregrin PE4283 RF UltraCMOS switches with a single-pin CMOS logic control input, a 1.5 $\mathrm{kV}$ ESD tolerance, a low insertion loss of $0.65 \mathrm{~dB}$ at the reference frequency, an isolation of $33.5 \mathrm{~dB}$ between the output ports, a $+3 \mathrm{~V}$ supply input and an operation band ranging from DC to $4 \mathrm{GHz}$. The study of both radiation patterns and current consumption has been performed through the use of STM32W-EXT WSN boards with a 32 bit ARM micropro- 


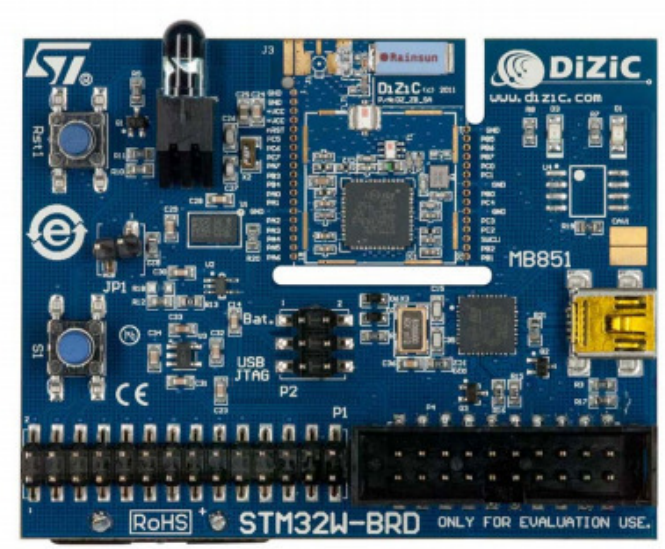

Fig 5. The STM32W-EXT WSN mote used for the experimental measurement.

cessor and an IEEE 802.15.4-compliant transceiver (see Fig. 5).

\section{A. Antenna characterization}

In order to characterize the antenna from an electromagnetic point of view, the reflection coefficient and the radiation pattern have been simulated and measured by varying the feeding configurations. In particular, the entire RF structure has been firstly modeled with the full-wave simulator CST- Microwave Studio [19] where the RF switches have been taken into account by means of proper equivalent circuits according to the datasheet. Return loss and radiation properties have been consequently calculated.

As described above, the proposed antenna presents eight switchable directional patterns, four of which, due to the feeding of Ant1-Ant2, Ant3-Ant4, Ant5-Ant6 and Ant7Ant8 elements, are determined by the same feeding configuration (Conf 1) whereas the other four, due to the feeding of Ant1-Ant3, Ant3-Ant5, Ant5-Ant7 and Ant7-Ant1 elements, are determined by a different configuration (Conf 2). For such a reason, without loss of generality, only data referred to Conf 1 and Conf 2 will be discussed hereafter.

In Fig. 6 and Fig. 7, the measured return loss obtained through a Vector Network Analyzer (VNA) Agilent 3444/7 is compared with the simulated results showing a very good agreement. In particular, both Conf 1 and Conf 2 exhibit an appreciable impedance matching with an observed return loss smaller than $-10 \mathrm{~dB}$ within the bandwidth of interest.

In Fig. 8 and Fig 9, the simulated radiation patterns in the azimuth $\left(\varphi=0^{\circ}\right)$, are reported in order to better appreciate the capability of the proposed antenna to correctly orientate the beam when varying the switch configuration. In particular, Fig. 8 is referred to the feeding of the L-shaped antenna element Ant1-Ant2 (belonging to Conf 1 scheme), and Fig. 9 to the feeding of Ant1-Ant3 (belonging to Conf 2 scheme), resulting in two adjacent radiation patterns separated by an angle of 30 degrees. The obtained main lobe magnitude for Conf 1 and Conf 2 are respectively $5.09 \mathrm{dBi}$ and $4.85 \mathrm{dBi}$, the side lobe levels are respectively $-15 \mathrm{~dB}$ and $-14 \mathrm{~dB}$ and
TABLE I.

Detailed Parameters of Designed Antenna

\begin{tabular}{|c|c|c|c|}
\hline Parameters & Value $[\mathbf{m m}]$ & Parameters & Value [mm] \\
\hline$\varepsilon_{r}$ & 4,7 & $\mathrm{~d}$ & 100 \\
\hline $\mathrm{h}$ & 0,8 & $\mathrm{~L}_{1}$ & 34 \\
\hline $\mathrm{e}$ & 1,5 & $\mathrm{~L}_{2}$ & 29 \\
\hline $\mathrm{f}$ & 1 & $\mathrm{k}$ & 4 \\
\hline $\mathrm{l}$ & 18 & $\mathrm{c}$ & 6 \\
\hline $\mathrm{l}_{\mathrm{s}}$ & 4,3 & $\mathrm{r}$ & 12 \\
\hline $\mathrm{g}$ & 17,8 & $\mathrm{~s}$ & 1 \\
\hline $\mathrm{g}_{\mathrm{s}}$ & 6,1 & $\mathrm{n}_{1}$ & 4 \\
\hline $\mathrm{t}_{1}$ & 48 & $\mathrm{n}_{2}$ & 7,7 \\
\hline $\mathrm{t}_{2}$ & 11 & $\mathrm{i}$ & 39 \\
\hline $\mathrm{t}_{3}$ & 25 & $\mathrm{w}_{\mathrm{s}}$ & 0,8 \\
\hline $\mathrm{t}_{4}$ & 20 & $\mathrm{M}_{\mathrm{t}}$ & 0,04 \\
\hline $\mathrm{r}_{\mathrm{i}}$ & 1,3 & $\mathrm{r}_{\mathrm{d}}$ & 4 \\
\hline
\end{tabular}

the cross-polarization levels are respectively $-21 \mathrm{~dB}$ and -20 dB. Moreover the Half power beam width (HPBW) is nearly 70 degrees in the azimuth plane for both configurations, according to the proposed design specifications. As can be seen, the proper functioning of the proposed switched-beam antenna, in terms of overlapping area and beam width, are demonstrated.

As further validation, in order to accurately characterize the radiation properties of the proposed antenna, several tests with WSN nodes operating in the ISM band have been performed. In particular, two STM32W108B-KEXT devices have been used, one connected to the proposed antenna and statically positioned in the middle of a $50 \times 50 \mathrm{~m}^{2}$ area, and the other, with a standard omnidirectional configuration, used to measure the number of packets received in different points of the same area (see Fig. 10). For each radiator, the diagram individuating the portion of the area where more than $95 \%$ of the sent packets is correctly received corresponds to the related actually covered area. As shown in Fig. 11 the measurement points are disposed on concentric circumferences with a minimum radius of $10 \mathrm{~m}(\mathrm{R} 1)$ and a maximum radius of $45 \mathrm{~m}(\mathrm{Rn})$ with an angular distance of $10^{\circ}$. In particular, Fig. 12 and 13 show the obtained diagrams for the two feeding configurations previously introduced (blue curve) and for the canonical dipole (green curve); black dots represent measurements points (see Fig. 11 for the experimental setup). In each case, the lower emitted power has been considered. As expected, it can be observed the proper functioning of the proposed switchedbeam antenna, that guarantees a longer working range than the half-wavelength dipole, which is, as expected, substantially omnidirectional in the azimuth plane. Alternatively, a lower power could be radiated to guarantee the same working distance. Moreover, as desired, coverage areas associated to the two feeding configurations guarantee a suitable overlapping area and a beam width compatible with the simulated one. In fact, despite conceptually different, the behaviour of the radiation patterns of Figs. 8 and 9 can be com- 


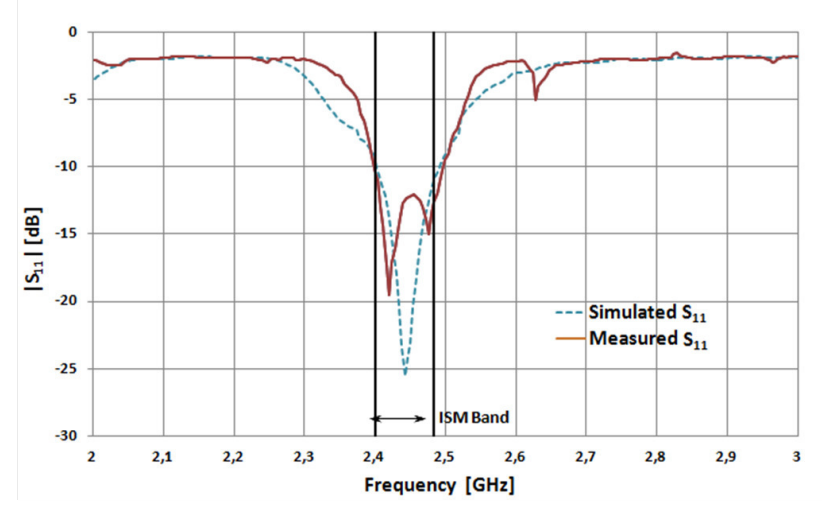

Fig 6. Simulated and measured reflection coefficient for the Conf 1 feeding configuration.

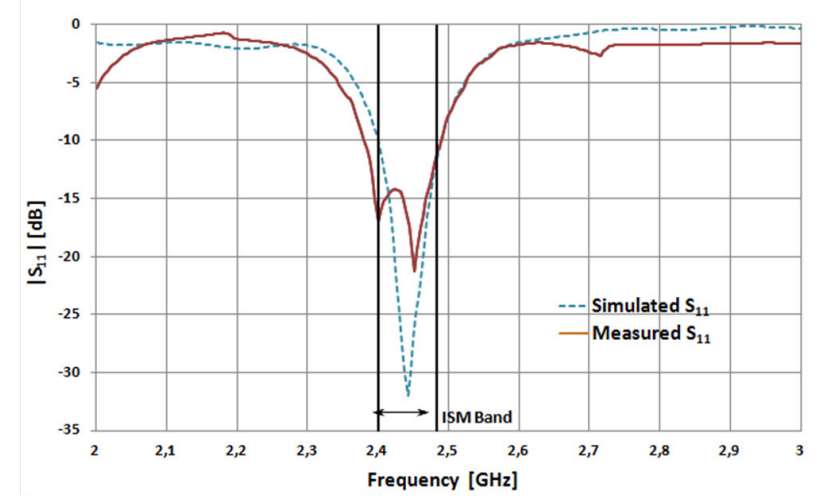

Fig 7. Simulated and measured reflection coefficient for the Conf 2 feeding configuration.

pared with those of the diagrams of Figs. 12 and 13. A substantial agreement can be observed for both feeding configurations previously introduced.

\section{B. Power consumption}

Several tests have been performed in order to accurately measure the power saving performance of the proposed antenna system. Similarly to the radiation patterns measurement, RSSI values estimated by STM32W-EXT WSN boards connected to the proposed antenna have been used also in this case. As previously explained, when the proposed antenna is used in place of the almost omnidirectional antenna of a mote, greater energy efficiency can be obtained. In order to estimate the power saving, the lower transmission power level $P_{t l}$ to be set on the mote equipped with the proposed antenna, with respect to a canonical mote, must be calculated. For this purpose, a study on the received power varying the distance between the motes has been conducted. For such a purpose, it is considered that the most realistic way to correlate the RSSI to the distance is to use the LogNormal Shadowing Model (LNSM) [20]-[22] which is able to predict the signal path loss in both indoor and outdoor conditions. This model is an extension particularly suitable for WSN context of the more general log-distance path loss

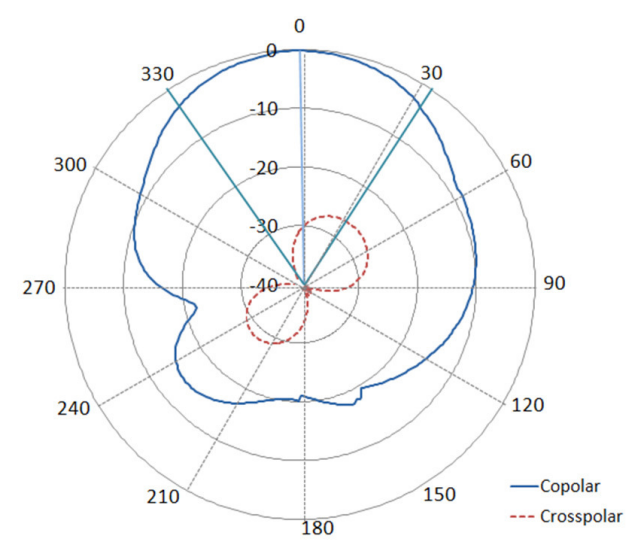

Phi / Degree vs. dB

Fig 8. Simulated radiation patterns for the Conf 1 feeding configuration in the azimuth plane.

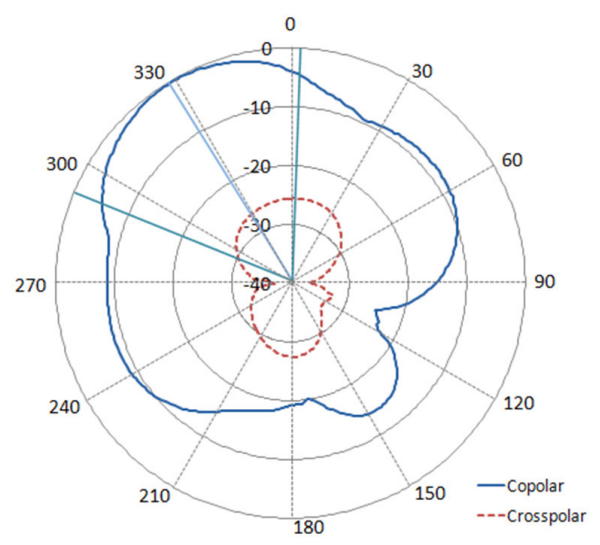

Phi / Degree vs. dB

Fig 9. Simulated radiation patterns for the Conf 1 feeding configuration in the azimuth plane.

model described in [20]. Indeed, this last does not consider that the measured power values can be very different from the predicted average ones, as the reflections caused by irregularities of the surrounding environment result in a variance of the measured values. LNSM states that the path loss follows a log-normal distribution (normal in $\mathrm{dB}$ ) due to the phenomenon called log-normal shadowing. Therefore the received power have a Gaussian distribution with zero mean and can be expressed as:

$$
P_{r}(d) \sim N\left(\overline{P_{r}(d), \sigma^{2}}\right)[d B m]
$$

where $P_{r}(d)$ is the average received power and $\sigma^{2}$ is the variance related to the effects of reflections. According to LNSM the equation (1) can be rewritten as: 


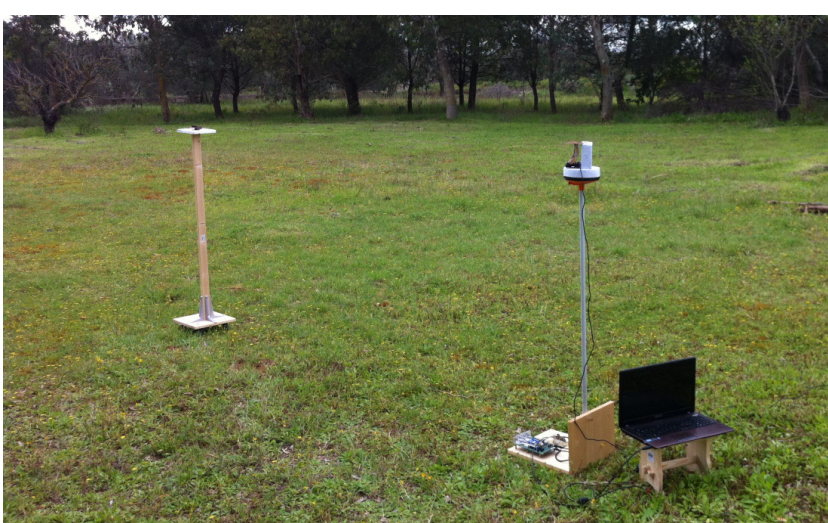

Fig 10. Measurement setup for the evaluation of the models and the diagrams of the average RSSI.

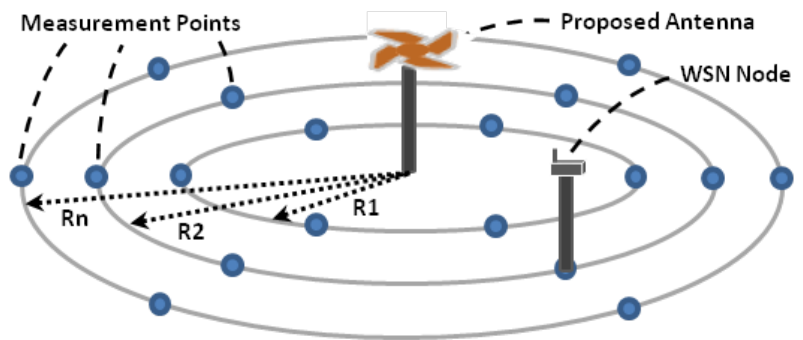

Fig 11. Experimental setup used for the evaluation of the diagrams of the coverage areas.

$$
\overline{P_{r}(d)}=\overline{P_{r}\left(d_{0}\right)}-10 n \log _{10}\left(\frac{d}{d_{0}}\right)+X_{\sigma}[d B m]
$$

where $P_{r}\left(d_{0}\right)$ is the power received at a reference distance $d_{0}$ that must be appropriate to the measurement context (in this case we selected a reference distance equal to $10 \mathrm{~m}$ ), $n$ is the path loss exponent that indicates how the received signal degrades in relation to the distance and depends on the electromagnetic characteristics of the surrounding environment, $X_{\sigma}$ is a normal random variable with zero mean.

To obtain a relationship between the received power and the distance between motes, it is necessary to estimate $n$ and $X_{\sigma}$; in practice, they are calculated from the measured power values at various distances, using the linear regression model:

$$
\overline{P_{r}(d)}=\alpha+\beta \log _{10}(d)[d B m]
$$

where $\alpha=P_{r}(10)+10 n+X_{\sigma}$ and $\beta=-10 n$. The path loss model of equation (3) can be used to estimate the distance between the transmitting and receiving mote as follows:

$$
d=10^{\frac{\overline{P_{r}(d)}-\alpha}{\beta}}[\mathrm{dBm}]
$$

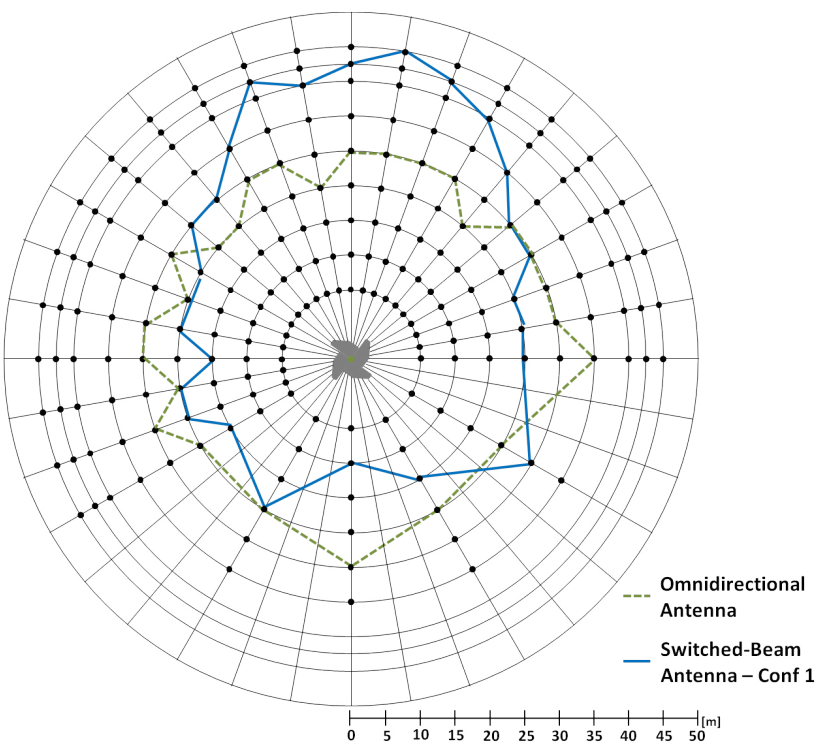

Fig 12. Diagram representing the regions where the WSN node in Conf 1 mode correctly receive more than $95 \%$ of packets.

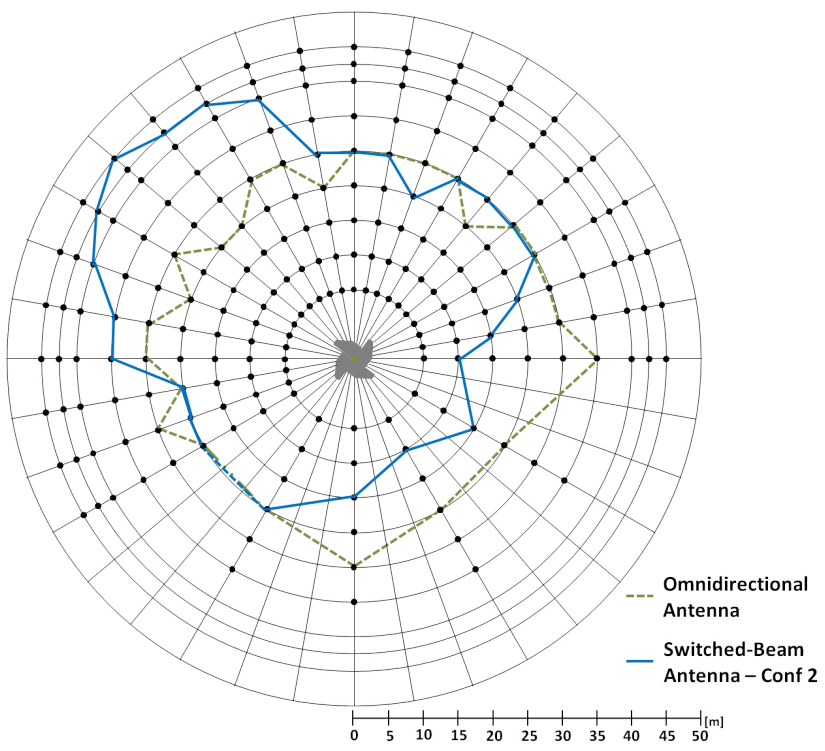

Fig 13. Diagram representing the regions where the WSN node in Conf 2 mode correctly receive more than $95 \%$ of packets.

In order to calculate the $\alpha$ and $\beta$ parameters, and accordingly $n$ and $X_{\sigma}$, several tests have been performed by varying both transmission power and distance and positioning the motes at an height of $1.5 \mathrm{~m}$. In particular, two kinds of tests have been performed in Line Of Sight (LOS) condition with surrounding obstacles. The former kind, named $\mathrm{T} 1$, has been performed using two motes equipped with a dipole antenna and setting the level $P_{t l}$ to $+3 \mathrm{dBm}$. Vice versa, the latter kind of tests, named $\mathrm{T} 2$, has been conducted using the proposed antenna and varying $P_{t l}$. In Fig. 14, the obtained results related to $\mathrm{T} 1$ and $\mathrm{T} 2$ tests, this last with $P_{t l}$ to $-3 \mathrm{dBm}$, 


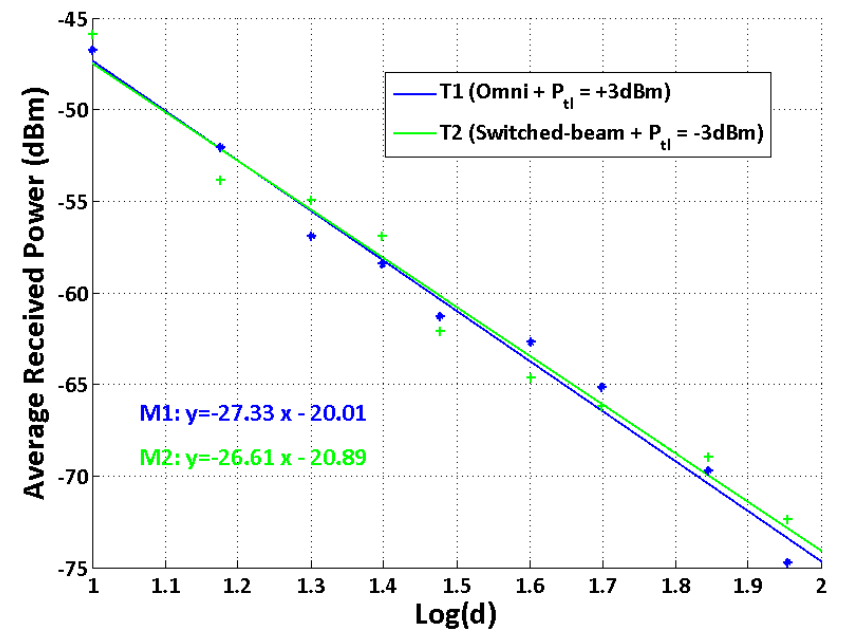

Fig 14. Average received power versus $\log (\mathrm{d})$ for the four tests performed.

are reported. As can be seen, despite the strong difference in terms of emitted power, the two curves are in very good agreement; moreover, it is worth observing that measured RSSI values exhibits a linear dependence with $\log (\mathrm{d})$. By relating the linear regression model in equation (3) with the experimental measurements performed, the parameters $n$ and $X_{\sigma}$ have been determined and reported in Table II.

Fig. 15, shows the standard deviations in RSSI measurements at each distance for the T1 and T2 tests. As can be expected, the deviation in RSSI measurements increases with the distance between the nodes.

Once the parameters $n$ and $X_{\sigma}$ are known, $\alpha$ and $\beta$ parameters can be straightforwardly be calculated and used in equation (4) to obtain the distance as a function of the received power. In Table 3 the estimated distance and the estimation error for the two tests considered are reported. In particular, Fig. 16 shows the comparison between the measured and estimated distances; the closeness of the curves proves the accuracy of the found models.

The presented results confirm the appropriateness of the proposed switched-beam antenna system as hardware element enabling new power-efficient WSNs. Indeed, as shown in Fig 14, the model M1 and model M2 are very similar. This is a crucial result because it allows a fair comparison in terms of power saving when the proposed antenna is used in place of the canonical dipole. Indeed, it is clear that, despite the different Ptl values, T1 and T2 configurations determine the same mote performance.

\section{Conclusions}

This work proposes a switched-beam antenna particularly suitable for WSN applications. It is composed of four arrays of two L-shaped quarter-wavelength slot antenna elements arranged in a symmetrical planar structure. According to the context-dependent needs of the WSN, one among eight different radiation patterns can be selected by means of properly designed control lines. The antenna system has been

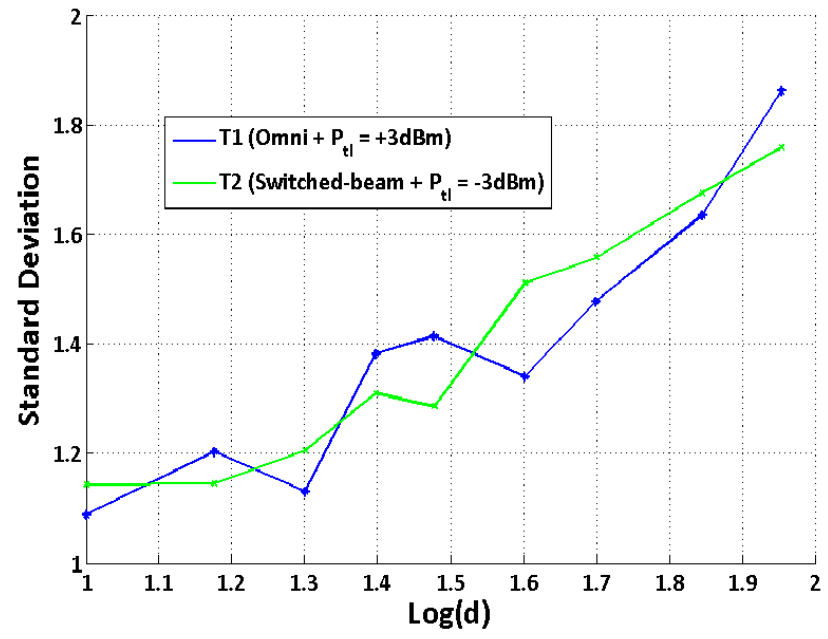

Fig 15. The standard deviations in RSSI measurements for T1 and T2

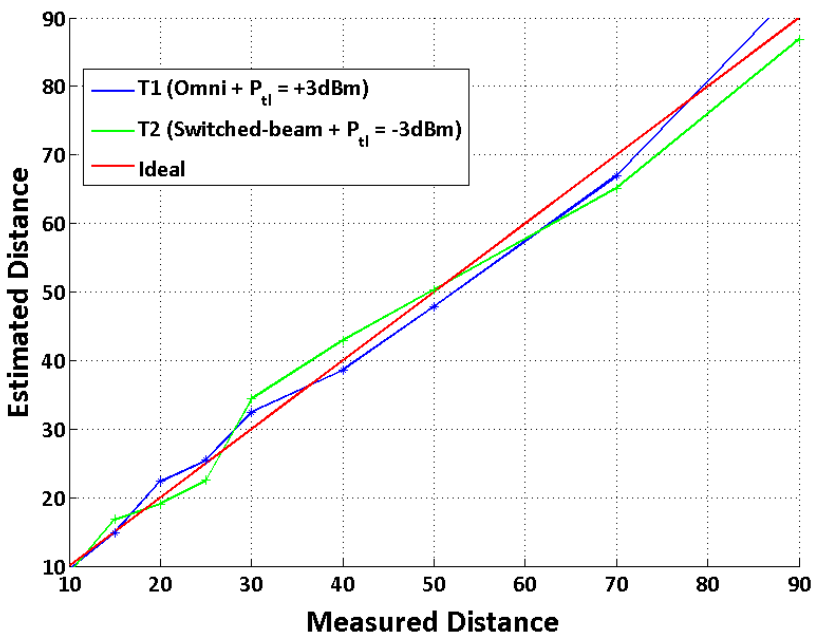

Fig 16. Measured versus estimated distance for the four models.

tests versus $\log (\mathrm{d})$.

rigorously characterized and has been then extensively tested in place of a canonical antenna mounted on a commercial WSN mote. The obtained and discussed results are really encouraging. Indeed, in spite of a very appreciable compactness and cost-effectiveness, the proposed switchedbeam antenna guarantees a considerable power saving if compared with traditional and almost omnidirectional WSN mote antennas.

\section{REFERENCES}

[1] G. Anastasi, M. Conti, M. Francesco, A. Passarella, "Energy conservation in wireless sensor networks: A survey," $A d$ Hoc Networks, vol. 7, no. 3, pp. 537-568, May 2009, http://dx.doi.org/10.1016/j.adhoc.2008.06.003

[2] G. Girban, M. Popa, "A glance on WSN lifetime and relevant factors for energy consumption," in Computational Cybernetics and Technical Informatics (ICCC-CONTI), 2010 International Joint Conference on, May 2010, pp. 523-528, http://dx.doi.org/10.1109/ICCCYB.2010.5491217 
TABLE II

Error of Estimated Distance

\begin{tabular}{|c|c|c|c|c|}
\hline $\begin{array}{c}\text { Measured } \\
\text { Distance } \\
{[\mathbf{m}]}\end{array}$ & $\begin{array}{c}\text { Estimated } \\
\text { Distance } \\
\mathbf{( T 1 )} \\
{[\mathbf{m}]}\end{array}$ & $\begin{array}{c}\text { Estimated } \\
\text { Distance } \\
\mathbf{( T 2 )} \\
\mathbf{[ m ]}\end{array}$ & $\begin{array}{c}\text { Error (T1) } \\
\mathbf{[ m ]}\end{array}$ & $\begin{array}{c}\text { Error (T2) } \\
\mathbf{[ m ]}\end{array}$ \\
\hline 10 & 9,51 & 8,92 & 0,49 & 1,08 \\
\hline 15 & 14,88 & 16,81 & 0,12 & 1,81 \\
\hline 20 & 22,39 & 19,05 & 2,39 & 0,95 \\
\hline 25 & 25,46 & 22,54 & 0,46 & 2,46 \\
\hline 30 & 32,43 & 34,42 & 2,43 & 4,42 \\
\hline 40 & 38,59 & 42,95 & 1,41 & 2,95 \\
\hline 50 & 47,9 & 50,35 & 2,1 & 0,35 \\
\hline 70 & 66,91 & 65,12 & 3,09 & 4,88 \\
\hline 90 & 94,46 & 86,91 & 4,46 & 3,09 \\
\hline
\end{tabular}

[3] Y. Chen, Q. Zhao, "On the Lifetime of Wireless Sensor Networks, " IEEE Communications Letters, Vol. 9, no. 11, pp. 976-978, Nov. 2005, http://dx.doi.org/10.1109/LCOMM.2005.11010

[4] L. Anchora, A. Capone, V. Mighali, L. Patrono, F. Simone, "A nove MAC scheduler to minimize the energy consumption in a Wireless Sensor Network," Ad Hoc Networks, vol. 16, pp. 88-104, May 2014, http://dx.doi.org/10.1016/j.adhoc.2013.12.002

[5] L. Catarinucci, R. Colella, G. Del Fiore, L. Mainetti, V. Mighali, L. Patrono, M.L. Stefanizzi, "A cross-layer approach to minimize the energy consumption in wireless sensor networks, " International Journal of Distributed Sensor Networks, vol. 2014, 2014, http://dx.doi.org/10.1155/2014/268284

[6] D. Alessandrelli, L. Mainetti, L. Patrono, G. Pellerano, M. Petracca, M.L. Stefanizzi, "Performance evaluation of an energy-efficient MAC scheduler by using a test bed approach," Journal of Communications Software and Systems, vol. 9, no. 1, pp. 84-96, Mar. 2013.

[7] G. Giorgetti, A. Cidronali, S.K.S. Gupta, G. Manes, "Exploiting LowCost Directional Antennas in 2.4 GHz IEEE 802.15.4 Wireless Sensor Networks", in Proc. 10th European Conference on Wireless Technology, Oct. 2007, pp. 217-220.

[8] S. Zhang, G. H. Huff, and J. T. Bernhard, "A pattern reconfigurable microstrip parasitic array," IEEE Trans. Antennas Propag., vol. 52, no. $10, \quad$ pp. 2773-2776, 2004 , http://dx.doi.org/10.1109/ECWT.2007.4403985

[9] M.-I. Lai, T.-Y. Wu, J.-C. Hsieh, C.-H. Wang, S.-K. Jeng, "Pattern Reconfigurable Antenna for a Wireless Sensor Network Sink Node," in Proc. Asia-Pacific Microwave Conference (APMC), Dec. 2010, pp. 2021-2024.
[10] D.-C. Chang, B.-H. Zeng, J.-C. Liu, "Reconfigurable angular diversity antenna with quad corner reflector arrays for $2.4 \mathrm{GHz}$ applications," IET Microw. Antennas Propag., vol. 3, no 3, pp. 522-528, Apr. http://dx.doi.org/2009, 10.1049/iet-map.2008.0119

[11] L. Ming-Iu, W. Tzung-Yu, H. Jung-Chin, W. Chun-Hsiung, J. ShyhKang, "Compact Switched-Beam Antenna Employing a Four-Element Slot Antenna Array for Digital Home Applications, "IEEE Trans. Antennas Propag., vol. 56 , no.9, pp 2929-2936, Sept. 2008. http://dx.doi.org/10.1109/TAP.2008.928775

[12] L. Catarinucci, S. Guglielmi, L. Patrono, and L. Tarricone, "Switchedbeam antenna for wireless sensor network nodes," Progress in Electromagnetics Research C, vol. 39, pp. 193-207, 2013, http://dx.doi.org/10.2528/PIERC13030707

[13] L. Catarinucci, S. Guglielmi, L. Mainetti, V. Mighali, L. Patrono, M.L. Stefanizzi, L. Tarricone, "An energy-efficient MAC scheduler based on a switched-beam antenna for wireless sensor networks," Journal of Communications Software and Systems, vol. 9, no. 2, pp. 117-127, Jun. 2013.

[14] L. Catarinucci, S. Guglielmi, R. Colella, L. Tarricone, "Compact switched-beam antenna enabling novel power-efficient wireless sensor network," IEEE Sensors J., vol. PP, no 99, pp. 1-9, May 2014, http://dx.doi.org/10.1109/JSEN.2014.2326971

[15] S. I. Latif, L. Shafai, and S. K. Sharma, "Bandwidth enhancement and size reduction of microstrip slot antennas," IEEE Trans. Antennas Propag., vol. 53, no. 3, pp. 994-1003, Mar. 2005, http://dx.doi.org/10.1109/TAP.2004.842674

[16] S. K. Sharma, L. Shafai, and N. Jacob, "Investigate of wide-band microstrip slot antenna," IEEE Trans. Antennas Propag., vol. 52, no. 3, pp. 865-872, Mar. 2004, http://dx.doi.org/10.1109/TAP.2004.825191

[17] L. Catarinucci, R. Colella, and L. Tarricone, "Smart Prototyping Techniques for UHF RFID Tags: Electromagnetic Characterization and Comparison with Traditional Approaches," Progress In Electromagnetics Research, Vol. 132, pp 91-111, Sep. 2012. http://dx.doi.org/10.2528/PIER12080708

[18] L. Catarinucci, R. Colella, L. Tarricone, "Prototyping Flexible UHF RFID Tags Through Rapid and Effective Unconventional Techniques: Validation on Label-Type Sensor-Tag," IEEE 2012 International Conference on RFID -Technologies and Applications (RFID - TA), $\mathrm{p}$. 176-181, Nice, Nov. 2012, http://dx.doi.org/10.1109/RFIDTA.2012.6404506

[19] CST - Computer Simulation Technology. [Online], https://www.cst.com, (last accessed: March. 2014)

[20] T.S. Rappaport, Wireless Communications: Principles and Practice, (2nd Edition), Prentice Hall, New York, Jan. 2007.

[21] R. Al Alawi, "RSSI based location estimation in wireless sensors networks", in Proc. 17th IEEE International Conference on Networks (ICON), Dec. 2007, pp. 118-122, http://dx.doi.org/10.1109/ICON.2011.6168517

[22] P. Kumar, L. Reddy, and S. Varma, "Distance measurement and error estimation scheme for rssi based localization in wireless sensor networks," in Proc IEEE Conference on Wireless Communication and Sensor Networks (WCSN), Dec. 2009, pp. 1-4, http://dx.doi.org/10.1109/WCSN.2009.5434802 